\title{
Mean-variance hedging in large financial markets*
}

\author{
Luciano CAMPI ${ }^{\dagger}$
}

\begin{abstract}
We consider a mean-variance hedging (MVH) problem for an arbitrage-free large financial market, i.e. a financial market with countably many risky assets modelled by a sequence of continuous semimartingales. By using the stochastic integration theory for a sequence of semimartingales developed in De Donno and Pratelli [6], we extend the results about change of numéraire and MVH of Gourieroux, Laurent and Pham [12] to this setting. We also consider, for all $n \geq 1$, the market formed by the first $n$ risky assets and study the solutions to the corresponding $n$-dimensional MVH problem and their behaviour when $n$ tends to infinity.
\end{abstract}

Key words: hedging, large financial market, stochastic integral for a sequence of semimartingales, numéraire, artificial extension method.

MS Classification (2000): 91B28, 60H05, 60G48

\section{Introduction}

In this paper we study a quadratic hedging problem for a future stochastic cash flow $F$, delivered at time $T$, in a non necessarily complete large financial market.

Let us consider first a market consisting of $n+1$ primitive assets $X=\left\{S^{0}, S\right\}$ : one bond with price process $S_{t}^{0}=\exp \left(\int_{0}^{t} r_{s} d s\right)$ and $n$ risky assets whose price process is a continuous $n$-dimensional semimartingale $S=\left(S^{1}, \cdots, S^{n}\right)$. A criterion for determining a "good" hedging strategy is to solve the mean-variance hedging (MVH) problem introduced by Föllmer and Sondermann [11]:

$$
\min _{\vartheta \in \Theta} \mathbb{E}\left[F-V_{T}^{x, \vartheta}\right]^{2}
$$

where

$$
V_{T}^{x, \vartheta}=S_{T}^{0}\left(x+\int_{0}^{T} \vartheta_{t} d\left(S / S^{0}\right)_{t}\right)
$$

${ }^{*}$ This research has been partially supported by the EU HCM-project Dynstoch (IMP, Fifth Framework Programme).

${ }^{\dagger}$ CEREMADE, Université Paris Dauphine, Place du Maréchal de Lattre de Tassigny, 75775 Paris Cedex 16. E-mail: campi@ceremade.dauphine.fr.

${ }^{\ddagger}$ I would like to thank Huyên Pham for fruitful suggestions and Marc Yor for his remarks. I am also grateful to Marzia De Donno and Maurizio Pratelli for their interest in this work. 
is the terminal value of a self-financed portfolio in the primitive assets, with initial investment $x$ and quantities $\vartheta$ invested in the risky assets.

This problem has been solved by Föllmer and Sondermann [11] and Bouleau and Lamberton [3] in the martingale case and, with different methods, by Gourieroux, Laurent and Pham [12] (abbr. GLP) and Rheinländer and Schweizer [21] in the semimartingale case under more or less restrictive conditions.

Here we focus on the GLP approach, which consists in adding a suitably chosen numéraire as an asset to trade in and changing the probability measure. The numéraire is chosen in such a way that the set of attainable contingent claims in the extended market remains unchanged. This procedure leads to considering a simpler quadratic hedging problem where the new prices process is a martingale so giving an explicit description of the optimal trading strategy for the original MVH-problem.

In this paper we seek to extend GLP's approach to a large financial market, i.e. a market with one bond and countably many risky assets. We recall that no-arbitrage in large financial markets has been studied for the first time in a rigorous way by Kabanov and Kramkov [14, 15] in the complete case and by Klein and Schachermayer [16] in the incomplete case. More recently, Bjork and Näslund [2] and De Donno [4] have investigated the completeness of some special models, where the assets prices dynamics are driven by one Brownian motion (common to all assets) and countably many independent Poisson processes (one for each asset).

In order to extend the GLP artificial extension method to an infinite-dimensional setting, one has to use a stochastic integration (SI) theory with respect to a sequence of semimartingales, i.e. with respect to a semimartingale taking values in the space $\mathbb{R}^{\mathbb{N}}$ of all real sequences, which is much more delicate to use than the vectorial one. Mikulevicius and Rozovskii [19] developed a SI theory for martingales taking values in a topological vector space (see also De Donno [4] and De Donno et al. [5] for financial applications). More recently, De Donno and Pratelli [6] have proposed a stochastic integral for a sequence of semimartingales, generalizing the SI theory by Mikulevicius and Rozovskii in this particular case. We will use their construction for making our MVH problem meaningful.

This paper is organized as follows. In Section 2, we recall some basic facts on stochastic integration with respect to a sequence of semimartingales and obtain an infinite-dimensional version of the Galtchouk-Kunita-Watanabe (abbr. GKW) decomposition theorem. In Section 3 , we define the good set of trading strategies and we show that the set of all attainable contingent claims is closed in $L^{2}(P)$. In Section 4 we show the invariance property for the set of all attainable claims. In Section 5, we show how to extend the artificial extension method to our setting and in Section 6 we consider the finite-dimensional MVH problems, corresponding to consider only the market formed by the bond and the first $n$ risky assets, and we show that the sequence of solutions of these finite-dimensional problems converges to the solution of the original one (1). 


\section{Some preliminaries on stochastic integration with respect to a sequence of semimartingales}

In this section we will follow very closely the treatment of the stochastic integration for countably many semimartingales, that the reader can find in De Donno and Pratelli [6].

Let $(\Omega, \mathcal{F}, P)$ be a probability space with a filtration $\mathbb{F}=\left\{\mathcal{F}_{t}, t \in[0, T]\right\}$ satisfying the usual conditions of right-continuity and $P$-completeness, where $T>0$ is a fixed finite horizon. Letting $p \geq 1$, we will denote by $\mathcal{H}^{p}(P)$ the set of all real-valued martingales $M$ on the given filtered probability space, such that $M^{*}=\sup _{t \in[0, T]}\left|M_{t}\right| \in L^{p}(P)$ (see Jacod's book [13] for more details). Moreover, we denote by $\mathcal{S}(P)$ the space of real semimartingales, equipped with Emery's topology (see Emery [10]). $\mathcal{S}(P)$ is a complete metric space.

Let $S=\left(S^{i}\right)_{i \geq 1}$ be a sequence of semimartingales. We denote by $E$ the set of all real-valued sequences (i.e. $\mathbb{R}^{\mathbb{N}}$ ), endowed with the topology of pointwise convergence and by $E^{\prime}$ its topological dual, namely the space of all signed measures on $\mathbb{N}$ which have a finite support; each of them can be identified with a sequence with all but finitely many components equal to 0 . We will denote by $e^{i}$ the element of both $E^{\prime}$ and $E$ such that $e_{j}^{i}=\delta_{i, j}$ (where $\delta_{i, j}$ is the Dirac delta); $\langle\cdot, \cdot\rangle_{E^{\prime}, E}$ will denote the duality between $E^{\prime}$ and $E$.

We denote by $\mathcal{U}$ the set of not necessarily bounded operators on $E$ and, for all $h \in \mathcal{U}$, we denote by $\mathcal{D}(h)$ the domain of $h(\mathcal{D}(h) \subset E)$. We say that a sequence $\left(h^{n}\right) \subset E^{\prime}$ converges to $h \in \mathcal{U}$ if $\lim _{n} h^{n}(x)=h(x)$, for every $x \in \mathcal{D}(h)$.

We say that a process $\xi$ taking values in $\mathcal{U}$ is predictable if there exists a sequence $\left(\xi^{n}\right)$ of $E^{\prime}$-valued predictable processes, such that for all $(\omega, t)$, and for all $x \in \mathcal{D}\left(\xi_{t}(\omega)\right)$, one has $\xi_{t}(\omega)=\lim _{n} \xi_{t}^{n}(\omega)$.

Finally, a $E^{\prime}$-valued predictable process $\xi$ is called a simple integrand if it has the form $\xi=\sum_{i \leqslant n} \xi^{i} e^{i}=\left(\xi^{1}, \xi^{2}, \ldots, \xi^{n}, 0, \cdots\right)$, where $\xi^{i}$ are real-valued predictable bounded processes. One can define the stochastic integral of a simple integrand $\xi$ with respect to $S$ in the following obvious way:

$$
\int \xi d S=\int \sum_{i \leqslant n} \xi^{i} d S^{i}
$$

Let $\xi$ be a predictable $\mathcal{U}$-valued process. Following De Donno and Pratelli [6], we will say that $\xi$ is integrable with respect to $S$ if there exists a sequence $\left(\xi^{n}\right)$ of simple integrands such that:

1. $\xi^{n}$ converges to $\xi$ pointwise;

2. $\int \xi^{n} d S$ converges to a semimartingale $Y$ in $\mathcal{S}(P)$.

We call $\xi$ a generalized integrand and define $\int \xi d S:=Y$. Moreover, we denote by $L(S, \mathcal{U})$ the set of generalized integrands.

This notion of stochastic integral is well-defined and implies an infinite-dimensional extension of Memin's theorem (Théorème V.4 [18]), which states that the set of stochastic integrals with respect to a semimartingale is closed in $\mathcal{S}(P)$ : 
Theorem 1 (De Donno and Pratelli [6], Theorem 5.2) Let $S=\left(S^{i}\right)_{i \geqslant 1}$ be given a sequence of semimartingales and $\left(\xi^{n}\right)$ a sequence of generalized integrands such that $\left(\int \xi^{n} d S\right)$ is a Cauchy sequence in $\mathcal{S}(P)$. Then there exists a generalized integrand $\xi$ such that $\int \xi^{n} d S \rightarrow$ $\int \xi d S$ in $\mathcal{S}(P)$.

In the sequel, we will need an infinite-dimensional version of the GKW-decomposition for a sequence of continuous local martingales. For this reason, we briefly recall the Mikulevicius and Rozovskii [19] theory of SI for a sequence of locally square integrable martingales and show how to extend it to a sequence of continuous local martingales.

We assume that $S^{i}=M^{i} \in \mathcal{H}^{2}(P)$ for all $i \geqslant 1$. It is easy to see that there exist:

1. an increasing predictable real-valued process $\left(A_{t}\right)$ with $\mathbb{E}\left[A_{T}\right]<\infty$,

2. a family $C=\left(C^{i j}\right)_{i, j \geqslant 1}$ of predictable real-valued process, such that $C$ is symmetric and non-negative definite, in the sense that $C^{i j}=C^{j i}$ and $\sum_{i, j \leqslant l} x_{i} C^{i j} x_{j} \geqslant 0$, for all $l \in \mathbb{N}$, for all $x \in \mathbb{R}^{l}, d P d A$ a.s.,

such that

$$
\left\langle M^{i}, M^{j}\right\rangle_{t}(\omega)=\int_{0}^{t} C_{s, \omega}^{i j} d A_{s}(\omega) .
$$

Consider $C$ for fixed $(\omega, t)$ and assume for simplicity that $C$ is positive definite. The above Itô isometry makes it natural to define on $E^{\prime}$ a norm by setting:

$$
|x|_{E_{t, \omega}^{\prime}}^{2}=\left\langle x, C_{t, \omega} x\right\rangle_{E^{\prime}, E}=\sum_{i, j=1}^{\infty} x_{i} C_{t, \omega}^{i j} x_{j},
$$

where the sum contains a finite number of terms. The norm is induced by an obvious scalar product, which makes $E^{\prime}$ a pre-Hilbert space. This norm depends on $(\omega, t)$ : for simplicity, we omit $\omega$, but we keep $t$ in the notation and denote $E_{t}^{\prime}$ the space $E^{\prime}$ with the norm induced by $C_{t}$. $E_{t}^{\prime}$ is not necessarily complete, but we can take its completion $H_{t}^{\prime}$ which is a Hilbert space. $H_{t}^{\prime}$ is generically not included in $E$, hence the canonical injection from $E^{\prime}$ to $E$ cannot be extended to an injection from $H_{t}^{\prime}$ to $E$.

The following theorem is essentially due to Mikulevicius and Rozovskii [19] (see their Proposition 11, p. 145). Nonetheless we prefer to follow the formulation of this result given by De Donno and Pratelli [6], since it fits better into their more general theory of SI for a sequence of semimartingales, as introduced before.

Theorem 2 (De Donno and Pratelli [6], Theorem 3.1) Let $\xi$ be a $\mathcal{U}$-valued process such that:

1. $\mathcal{D}\left(\xi_{\omega, t}\right) \supset H_{\omega, t}$ for all $(\omega, t)$;

2. $\left.\xi_{\omega, t}\right|_{H_{\omega, t}} \in H_{\omega, t}^{\prime}$;

3. $\xi_{t}\left(C_{t} e_{n}\right)$ is predictable for all $n$; 
4. $\mathbb{E}\left[\int_{0}^{T}\left|\xi_{t}\right|_{H_{t}^{\prime}}^{2} d A_{t}\right]<\infty$.

Then, there exists a sequence $\xi^{n}$ of simple integrands, such that $\xi_{\omega, t}^{n}$ converges to $\xi_{\omega, t}$ in $H_{\omega, t}^{\prime}$ for all $(\omega, t)$ and $\int \xi^{n} d M$ is a Cauchy sequence in $\mathcal{H}^{2}(P)$. As a consequence, we can define the stochastic integral $\int \xi d M$ as the limit of the sequence $\int \xi^{n} d M$.

Remark 3 The set of all stochastic integrals $\int \xi d M$, with $\xi$ fulfilling the four conditions of Theorem 2, is a closed set in $\mathcal{H}^{2}(P)$ and coincides with the stable subspace generated by $M$ in $\mathcal{H}^{2}(P)$. It is an immediate extension of the analogous result in the finite-dimensional case.

When $M=\left(M^{i}\right)_{i \geqslant 1}$ is a sequence of continuous local martingales, it is quite easy to extend the previous construction. Indeed, from Dellacherie [9] (Théorème 2 and Théorème 3 , p. 743) we deduce that there exists a uniform localization for the sequence $\left(M^{i}\right)$, i.e. a sequence $\left(\tau_{n}\right)$ of stopping times such that $\tau_{n} \rightarrow T$ and $M_{\cdot \wedge \tau_{n}}^{i}$ is a bounded martingale for all $i \geqslant 1$.

This property allows us to define by localization, in the usual way, a stochastic integral with respect to $M$ and for all $\mathcal{U}$-valued processes $\xi$ such that:

1. $\mathcal{D}\left(\xi_{\omega, t}\right) \supset H_{\omega, t}$ for all $(\omega, t)$;

2. $\left.\xi_{\omega, t}\right|_{H_{\omega, t}} \in H_{\omega, t}^{\prime}$;

3. $\xi_{t}\left(C_{t} e_{n}\right)$ is predictable for all $n$;

4. $\int_{0}^{T}\left|\xi_{t}\right|_{H_{t}^{\prime}}^{2} d A_{t}<\infty P$-a.s..

Finally, again by using Dellacherie's uniform localization, it is easy to prove a GKWdecomposition in our setting:

Proposition 4 Let $M=\left(M^{i}\right)_{i \geqslant 1}$ be a sequence of continuous local martingales and $N$ be a real-valued local martingale. Then, there exists an integrand $\xi$ satisfying conditions 1.-4. as in Theorem 2, and a real-valued local martingale $L$ vanishing at zero and orthogonal to each $M^{i}$, such that

$$
N=N_{0}+\int \xi d M+L
$$

Proof. Apply the Dellacherie uniform localization and Remark 3 and proceed exactly as, for instance, in Jacod [13], Théorème (4.27) if $N$ is locally square-integrable. Otherwise, use the following argument by Ansel and Stricker [1] (D.K.W. cas 3): write $N$ as the sum $N=N^{c}+N^{d}$, where $N^{c}$ and $N^{d}$ are its continuous and purely discontinuous parts. $N^{d}$ is orthogonal to all continuous local martingales and $N^{c}$ is locally bounded and then it can be written as $N^{c}=\int \xi d M+U$ with $U$ orthogonal to $M$ and $\xi$ satisfying conditions 1 . to 4.. To conclude, it suffices to set $L=U+N^{d}$. 


\section{The market model}

Let $(\Omega, \mathcal{F}, P)$ be a probability space with a filtration $\mathbb{F}=\left\{\mathcal{F}_{t}, t \in[0, T]\right\}$ satisfying the usual conditions of right-continuity and $P$-completeness, where $T>0$ is a fixed finite horizon. We also assume that $\mathcal{F}_{0}$ is trivial and $\mathcal{F}_{T}=\mathcal{F}$.

In this market model agents can trade in countably many primitive assets whose prices are modelled by a sequence of real valued processes $X=\left(S^{i}\right)_{i>0}$ : a bond price process $S_{t}^{0}=\exp \int_{0}^{t} r_{s} d s$, with $r$ a progressively measurable process interpreted as the instantaneous interest rate and such that $\left|\int_{0}^{T} r_{s} d s\right| \leq c$ for some constant $c>0$, and countably many risky assets, whose price processes $S^{i}$ are assumed to be continuous semimartingales.

We set

$$
\mathcal{M}_{2}=\left\{Q \ll P: \frac{1}{S_{T}^{0}} \frac{d Q}{d P} \in L^{2}(P), \text { every } S^{i} / S^{0} \text { is a } Q \text {-local martingale }\right\}
$$

and

$$
\mathcal{M}_{2}^{e}=\left\{Q \in \mathcal{M}_{2}: Q \sim P\right\} .
$$

Throughout the paper, we make the natural standing assumption:

$$
\mathcal{M}_{2}^{e} \neq \emptyset .
$$

This assumption is related to some kind of no-arbitrage condition (see, e.g., the seminal paper by Kreps [17]).

We denote by $\Theta$ the space of all generalized integrands $\vartheta \in L\left(S / S^{0}, \mathcal{U}\right)$ such that $\int_{0}^{T} \vartheta_{t} d\left(S / S^{0}\right)_{t} \in L^{2}(P)$ and for all $Q \in \mathcal{M}_{2}^{e} \int \vartheta d\left(S / S^{0}\right)$ is a $Q$-martingale.

Notice (see the discussion in De Donno [4], pp. 8-11) that in general one cannot define the value process of a trading strategy $\vartheta$ in the usual way: the expression $\vartheta_{t} \cdot\left(S / S^{0}\right)_{t}$ is not always well-defined. This is because $\vartheta_{t}$ takes values in the space $\mathcal{U}$ which is, in most cases, strictly bigger than $E^{\prime}$, and so we cannot use duality to define a product between the strategy $\vartheta$ and the price process. For this reason we will use the following:

Definition 5 For a trading strategy $\vartheta \in \Theta$ the value process of the corresponding selffinanced portfolio with respect to the primitive asset family $\left\{S^{0}, S\right\}$ and with initial value $x \in \mathbb{R}$ is given by

$$
V_{t}=V_{t}^{x, \vartheta}=S_{t}^{0}\left(x+\int_{0}^{t} \vartheta_{s} d\left(S / S^{0}\right)_{s}\right), \quad t \in[0, T] .
$$

Moreover we denote by

$$
G_{T}(x, \Theta):=\left\{V_{T}^{x, \vartheta}: \vartheta \in \Theta\right\} \subseteq L^{2}(P)
$$

the set of investment opportunities (or attainable claims) with initial value $x \in \mathbb{R}$.

The next proposition makes our MVH-problem meaningful, ensuring the existence of its solution $\theta^{*}$ and uniqueness of the corresponding optimal value process $V^{x, \vartheta^{*}}$. 
Proposition 6 The set $G_{T}(x, \Theta)$ is closed in $L^{2}(P)$.

Proof. Let $\vartheta^{n}$ be a sequence in $\Theta$ such that $S_{T}^{0}\left(x+\int_{0}^{T} \vartheta_{s}^{n} d\left(S / S^{0}\right)_{s}\right)$ converges in $L^{2}(P)$ to a random variable $V$. Take some $Q \in \mathcal{M}_{2}^{e}$ and set $Y_{t}^{n}:=\int_{0}^{t} \vartheta_{s}^{n} d\left(S / S^{0}\right)_{s}, t \in[0, T]$. Observe that the sequence of $Q$-martingales $\left(Y_{t}^{n}\right)_{t \in[0, T]}$ converges in $\mathcal{H}^{1}(Q)$ (see, e.g., Remark 2.2 . in Delbaen and Schachermayer [8]). Now, since convergence in $\mathcal{H}^{1}(Q)$ implies that in $\mathcal{S}(Q)$ (see Theorem 14 in Protter [20], p. 208) and Emery's topology is invariant under a change of an equivalent probability measure (see Théorème II.5 in Memin [18], p. 20), the sequence $\left(Y_{t}^{n}\right)_{t \in[0, T]}$ converges also in $\mathcal{S}(P)$. Now, thanks to Theorem 1 we can exhibit a generalized integrand $\vartheta \in L\left(S / S^{0}, \mathcal{U}\right)$ such that

$$
V=S_{T}^{0}\left(x+\int_{0}^{T} \vartheta_{s} d\left(S / S^{0}\right)_{s}\right) .
$$

The other two properties defining the set $\Theta$ are obviously satisfied also by the process $\vartheta$.

\section{Extending the GLP artificial extension method}

According to GLP, we define a numéraire as a self-financed portfolio with respect to the primitive assets family $X=\left\{S^{0}, S\right\}$, which is characterized by a trading strategy $a \in \Theta$ and a strictly positive value process $V(a)=V^{1, a}>0$ as in (7) with $V_{0}(a)=1$.

Remark 7 To avoid misunderstandings when comparing our results to GLP's, observe that we have denoted by "a" the strategy used as a numéraire, instead of the integrand in its exponential representation as in GLP.

To such a numéraire $a$ we can associate a new family of countably many assets consisting of this numéraire and the primitive assets. This assets family is called, as in GLP, a-extended assets family, its price process is given by $\{V(a), X\}$ while its price process renormalized in the new numéraire is $\{1, X(a)\}:=\{1, X / V(a)\}$.

Given a numéraire $a \in \Theta$, we define

$$
\mathcal{M}_{2}(a)=\left\{Q(a) \ll P: \frac{1}{V_{T}(a)} \frac{d Q(a)}{d P} \in L^{2}(P) \text {, every } X(a)^{i} \text { is a local } Q(a) \text {-martingale }\right\}
$$

and

$$
\mathcal{M}_{2}^{e}(a)=\left\{Q(a) \in \mathcal{M}_{2}(a): Q(a) \sim P\right\} .
$$

As in GLP Proposition 3.1, and with the same proof, we have the following characterization of the set $\mathcal{M}_{2}^{e}(a)$ of equivalent a-martingale measures in terms of the set $\mathcal{M}_{2}^{e}$ of equivalent martingale measures: 
Proposition 8 Let $a \in \Theta$ be a numéraire and $V(a)$ its value process. There is a one-to-one correspondence between $\mathcal{M}_{2}(a)$ (resp. $\mathcal{M}_{2}^{e}(a)$ ) and $\mathcal{M}_{2}$ (resp. $\left.\mathcal{M}_{2}^{e}\right): Q(a) \in \mathcal{M}_{2}(a)$ (resp. $\left.\mathcal{M}_{2}^{e}(a)\right)$ if and only if there exists $Q \in \mathcal{M}_{2}$ (resp. $\left.\mathcal{M}_{2}^{e}\right)$ such that

$$
\frac{d Q(a)}{d P}=\frac{V_{T}(a)}{S_{T}^{0}} \frac{d Q}{d P} .
$$

We denote by $\Phi(a)$ the space of trading strategies with respect to the $a$-extended assets family $\{V(a), X\}$, i.e. the set of all $\phi(a) \in L(X(a), \mathcal{U})$ such that $V_{T}(a) \int_{0}^{T} \phi_{t}(a) d X_{t}(a) \in$ $L^{2}(P)$ and $V(a) \int \phi(a) d X(a)$ is a local $Q(a)$-martingale for all $Q(a) \in \mathcal{M}_{2}^{e}(a)$.

For a trading strategy $\phi(a) \in \Phi(a)$ the value process of the corresponding self-financed portfolio with respect to the $a$-extended assets family $\{V(a), X\}$ and with initial value $x \in \mathbb{R}$ is given by

$$
V_{t}=V_{t}^{x, \phi(a)}=V_{t}(a)\left(x+\int_{0}^{t} \phi_{s}(a) d X_{s}(a)\right) \quad t \in[0, T]
$$

Furthermore,

$$
G_{T}(x, \Phi(a)):=\left\{V_{T}^{x, \phi(a)}: \phi(a) \in \Phi(a)\right\}
$$

denotes the set of terminal values of self-financed portfolios with respect to the $a$-extended assets family $\{V(a), X\}$ and with initial value $x$.

In the finite assets case (GLP, Proposition 3.2, p. 186-188) the artificial extension leaves invariant the investment opportunity set, and gives explicit expressions for the correspondences linking the investment opportunities sets. We briefly recall this result: let $a^{n}$ be a $n$-dimensional numéraire and $V\left(a^{n}\right)=V^{1, a^{n}}$ its value process,

- to a self-financed portfolio $\left(V^{n}, \vartheta^{n}\right)$ with respect to $\left\{S^{0}, \bar{S}^{n}\right\}$, corresponds the selffinanced portfolio $\left(V^{n}, \phi^{n}\left(a^{n}\right)\right)=\left(V^{n},\left(\eta^{n}\left(a^{n}\right), \vartheta^{n}\left(a^{n}\right)\right)\right)$ with respect to $\left\{V\left(a^{n}\right), S^{0}, S\right\}$ given by

$$
\eta_{t}^{n}\left(a^{n}\right)=\frac{V_{t}^{n}-\vartheta_{t}^{n} \bar{S}_{t}^{n}}{S_{t}^{0}} \quad \text { and } \quad \vartheta_{t}^{n}\left(a^{n}\right)=\vartheta_{t}^{n}, \quad t \in[0, T]
$$

- to a self-financed portfolio $\left(V^{n}, \phi^{n}\left(a^{n}\right)\right)=\left(V^{n},\left(\eta^{n}\left(a^{n}\right), \vartheta^{n}\left(a^{n}\right)\right)\right)$ w.r.t. $\left\{V\left(a^{n}\right), S^{0}, S\right\}$ corresponds the self-financed portfolio $\left(V^{n}, \vartheta^{n}\right)$ w.r.t. $\left\{S^{0}, \bar{S}^{n}\right\}$ given by

$$
\vartheta_{t}^{n}=\vartheta_{t}^{n}\left(a^{n}\right)+a_{t}^{n} \frac{V_{t}^{n}-\phi_{t}^{n}\left(a^{n}\right) \bar{X}_{t}^{n}}{V_{t}\left(a^{n}\right)}, \quad t \in[0, T] .
$$

Note that the above expressions involve some scalar products between strategies and price processes, which in our infinite-dimensional setting are not well-defined. This makes very difficult to find the infinite-dimensional analogues of the GLP-correspondences above. Nonetheless, the following proposition states their existence for our large financial market and, as a straightforward consequence, the invariance of the investment opportunities set under a change of numéraire.

Proposition 9 Let $a \in \Theta$ be a numéraire and $V(a)$ its value process. 
1. Let $\vartheta \in \Theta$ be a trading strategy with respect to the primitive assets family $\left\{S^{0}, S\right\}$ and let $V$ denote the value process of the corresponding self-financed portfolio. Then there exists a trading strategy $\phi(a) \in \Phi(a)$ with respect to the a-extended assets family $\{V(a), X\}$ with the same value process $V$.

2. Let $\phi(a)=(\eta(a), \vartheta(a)) \in \Phi(a)$ be a trading strategy with respect to the a-extended assets family $\{V(a), X\}$ and let $V$ denote the value process of the corresponding selffinanced portfolio. Then there exists a trading strategy $\vartheta \in \Theta$ with respect to the primitive assets family $\left\{S^{0}, S\right\}$ with the same value process $V$.

3. We have in particular that

$$
G_{T}(x, \Theta)=G_{T}(x, \Phi(a)) .
$$

Proof. 1. Let $\vartheta \in \Theta$ be a trading strategy with respect to the primitive assets family $\left\{S^{0}, S\right\}$ with value process $V=S^{0}\left(V_{0}+\int \vartheta d\left(S / S^{0}\right)\right)$. By the definition of SI for a sequence of semimartingales there exists a sequence $\vartheta^{n}$ of simple integrands w.r.t. $S / S^{0}$ such that $\int \vartheta^{n} d\left(S / S^{0}\right)$ converges in $\mathcal{S}(P)$ to $\int \vartheta d\left(S / S^{0}\right)$.

We associate to each approximating strategy $\vartheta^{n}$ a self-financed portfolio with respect to the primitive assets family, whose value process is given by

$$
V_{t}^{n}=S_{t}^{0}\left(V_{0}+\int_{0}^{t} \vartheta_{s}^{n} d\left(S^{n} / S^{0}\right)_{s}\right), \quad t \in[0, T] .
$$

By GLP, Proposition 3.2 (i), there exists a trading strategy $\phi^{n}(a)=\left(\eta^{n}(a), \vartheta^{n}(a)\right)$ given by (10) with $V^{n}(a)$ instead of $V^{n}\left(a^{n}\right)$ and with the same value process $V^{n}$, i.e.

$$
S_{t}^{0}\left(V_{0}+\int_{0}^{t} \vartheta_{s}^{n} d\left(S / S^{0}\right)_{s}\right)=V_{t}(a)\left(V_{0}+\int_{0}^{t} \phi_{s}^{n}(a) d X_{s}(a)\right), \quad t \in[0, T] .
$$

By the multidimensional version of Proposition 4 in Emery (1979), we have that

$$
\frac{S^{0}}{V(a)}\left(V_{0}+\int \vartheta^{n} d\left(S / S^{0}\right)\right) \rightarrow \frac{S^{0}}{V(a)}\left(V_{0}+\int \vartheta d\left(S / S^{0}\right)\right)
$$

in $\mathcal{S}(P)$, as $n \rightarrow \infty$, and so the sequence $\int \phi^{n}(a) d X(a)$ is convergent in $\mathcal{S}(P)$. Now, by the infinite-dimensional version of Memin's theorem (Theorem 1 ) there exists a generalized integrand $\phi(a) \in L(X(a), \mathcal{U})$ such that $V_{0}+\int \phi^{n}(a) d X(a) \rightarrow V_{0}+\int \phi(a) d X(a)$ in $\mathcal{S}(P)$, as $n \rightarrow \infty$, and obviously for all $t \in[0, T]$

$$
S_{t}^{0}\left(V_{0}+\int_{0}^{t} \vartheta_{s} d\left(S / S^{0}\right)_{s}\right)=V_{t}(a)\left(V_{0}+\int_{0}^{t} \phi_{s}(a) d X_{s}(a)\right) .
$$

Finally, by Proposition 8 and since $\vartheta \in \Theta$, the process $\int \phi(a) d X(a)$ is a local $Q(a)$ martingale for all $Q(a) \in \mathcal{M}_{2}^{e}(a)$, and also $V_{T}(a) \int_{0}^{T} \phi_{s}(a) d X_{s}(a) \in L^{2}(P)$, i.e. $\phi(a) \in \Phi(a)$. 
2. Let $\phi(a) \in \Phi(a)$ be a trading strategy with respect to the $a$-extended assets family $\{V(a), X\}$ with value process of the corresponding self-financed portfolio given by $V(a)\left(V_{0}+\right.$ $\left.\int \phi(a) d X(a)\right)$.

By definition of $\Phi(a)$, there exists a sequence of simple integrands $\phi^{n}(a)=\left(\eta^{n}(a), \vartheta^{n}(a)\right)$, with $\eta^{n}(a)$ real-valued, converging pointwise to $\phi(a)$ and such that

$$
\int \phi^{n}(a) d X(a) \rightarrow \int \phi(a) d X(a)
$$

in $\mathcal{S}(P)$ as $n \rightarrow \infty$.

Denote by $V^{n}$ the value process of the approximating self-financed portfolio corresponding to $\phi^{n}(a)$, i.e. $V^{n}=V(a)\left(V_{0}+\int \phi_{s}^{n}(a) d X(a)\right)$, and consider the following sequence of strategies $\vartheta^{n}$ with respect to $\left\{S^{0}, S\right\}$ defined by the GLP correspondence (11):

$$
\vartheta_{t}^{n}=\vartheta_{t}^{n}(a)+a_{t} \psi_{t}^{n}(a), \quad t \in[0, T],
$$

where

$$
\psi^{n}=\frac{V^{n}-\phi^{n}(a) \bar{X}^{n}}{V(a)} .
$$

We remark that the process $\vartheta^{n}$ takes values in $\mathcal{U}$. Now, if we proceed as in the second part of the proof of Proposition 3.2 in GLP (observe that, by definition of generalized integrand, $\phi^{n}(a)$ is bounded, which implies $\psi^{n}(a)$ locally bounded), we obtain

$$
d\left(V^{n} / S^{0}\right)_{t}=\psi_{t}^{n} d\left(V(a) / S^{0}\right)_{t}+\vartheta_{t}^{n}(a) d\left(S / S^{0}\right)_{t} .
$$

Being $d\left(V(a) / S^{0}\right)_{t}=a_{t} d\left(S / S^{0}\right)_{t}$ with $a \in L\left(S / S^{0}, \mathcal{U}\right)$, if we approximate $a$ by a sequence $a^{k}$ of simple integrands converging pointwise to $a$ and such that $\int a^{k} d\left(S / S^{0}\right) \rightarrow \int a d\left(S / S^{0}\right)$ in $\mathcal{S}(P)$, also the sequence $\int \psi^{n}(a) a^{k} d\left(S / S^{0}\right)$ converges in $\mathcal{S}(P)$ with $n$ fixed and $k$ tending to infinity and then, by Theorem 1 , there exists a generalized integrand $\zeta^{n}$ such that

$$
\psi_{t}^{n}(a) d\left(\frac{V(a)}{S^{0}}\right)_{t}=\zeta_{t}^{n} d\left(\frac{S}{S^{0}}\right)_{t},
$$

and moreover, since $\psi^{n}(a) a^{k}$ converges pointwise to $\psi^{n}(a) a, \zeta^{n}=\psi^{n}(a) a$. Furthermore

$$
S_{t}^{0}\left(V_{0}+\int_{0}^{t} \vartheta_{s}^{n} d\left(S / S^{0}\right)_{s}\right)=V_{t}(a)\left(V_{0}+\int_{0}^{t} \phi_{s}^{n}(a) d X_{s}(a)\right), \quad t \in[0, T] .
$$

Finally, by letting $n$ tend to infinity and by using the same argument (infinite-dimensional version of Memin's theorem) as in the previous part of the proof (after exchanging the rôles of $\vartheta^{n}$ and $\phi^{n}(a)$ ), one can easily show that there exists a strategy $\vartheta \in \Theta$, whose value process equals $V$. The proof of item 2 . is now complete.

3. This statement follows trivially from the first two items of this proposition.

We just mentioned that, since it is not possible in this setting to define a product between strategies and price processes, we are not able to find an explicit expression for the 
infinite-dimensional GLP correspondences. We only know that the two sets of strategies are related by the equality of their value processes, i.e. given a strategy $\vartheta$ (resp. $\phi(a)$ ) its corresponding strategy $\phi(a)$ (resp. $\vartheta$ ) satisfies the following equation:

$$
S_{t}^{0}\left(V_{0}+\int_{0}^{t} \vartheta_{s} d\left(S / S^{0}\right)_{s}\right)=V_{t}(a)\left(V_{0}+\int_{0}^{t} \phi_{s}(a) d X_{s}(a)\right), \quad t \in[0, T] .
$$

The previous proposition ensures the existence of a solution to this equation when $\vartheta$ (resp. $\phi(a))$ is fixed.

Remark 10 In Section 6, we will see that there exists a sequence of predictable trading strategies $\vartheta^{n, *}$, that both solve the MVH-problem arisen by considering only the first $n$ risky assets, and its value processes converge to the value process of $\vartheta^{*}$, solution to problem $(1)$, in $L^{2}(P)$ as $n$ tends to infinity.

\section{The MVH problem}

We would like to apply the artificial extension method introduced by GLP to the following "large" mean-variance hedging optimization problem:

$$
J(x, F):=\min _{\vartheta \in \Theta} \mathbb{E}\left[F-S_{T}^{0}\left(x+\int_{0}^{T} \vartheta_{t} d\left(S / S^{0}\right)_{t}\right)\right]^{2} .
$$

where $F \in L^{2}(P)$ and $x \in \mathbb{R}$ are fixed. In financial terms, given an $\mathcal{F}_{T}$-measurable contingent claim $F \in L^{2}(P)$, we are looking for a self-financed portfolio with respect to the primitive assets family $\left\{S^{0}, S\right\}$, with initial investment $x$, that minimizes the expected square of the hedging residual. By Proposition 6 there exists a solution $\vartheta^{*}=\vartheta^{*}(x, H)$ to the problem $(\mathcal{H}(x))$ called the optimal hedging strategy, leading to a unique optimal value process

$$
V_{t}^{*}=V_{t}^{*}(x, F)=S_{T}^{0}\left(x+\int_{0}^{T} \vartheta_{t}^{*}(x, F) d\left(S / S^{0}\right)_{t}\right) .
$$

The couple $\left(V^{*}, \vartheta^{*}\right)$ is called optimal hedging portfolio.

Let us consider the solution $\widetilde{a} \in \Theta$ to following optimization problem:

$$
\min _{\vartheta \in \Theta} \mathbb{E}\left[S_{T}^{0}\left(1+\int_{0}^{T} \vartheta_{t} d\left(S / S^{0}\right)_{t}\right)\right]^{2}
$$

which is a particular case of $(\mathcal{H}(x))$ for a zero cash flow $F=0$ and initial wealth $x=1$. The strategy $\widetilde{a} \in \Theta$ leads to a unique terminal wealth $V_{T}(\widetilde{a})=V_{T}^{1, \widetilde{a}}=S_{T}^{0}\left(1+\int_{0}^{T} \widetilde{a}_{t} d\left(S / S^{0}\right)_{t}\right)$.

Now, let us consider the variance-optimal martingale measure (abbr. VOMM) $\widetilde{P}$, defined as the solution to the dual quadratic problem of $(\mathcal{P})$ :

$$
\min _{Q \in \mathcal{M}_{2}} \mathbb{E}\left[\frac{1}{S_{T}^{0}} \frac{d Q}{d P}\right]^{2}
$$


It is well-known that under the assumption (6), such a measure $\widetilde{P}$ exists and belongs to $\mathcal{M}_{2}$.

By reproducing exactly the same arguments as in Section 4 of GLP, one can easily see that $V(\widetilde{a})>0$, so that we can use it as a numéraire called hedging numéraire, and that $\widetilde{P}$ is related to $\widetilde{a}$ by

$$
\frac{d \widetilde{P}}{d P}=\frac{V_{T}(\widetilde{a}) S_{T}^{0}}{\mathbb{E}\left[V_{T}(\widetilde{a}) S_{T}^{0}\right]}
$$

Following GLP, we will solve problem $(\mathcal{H}(x))$ by transforming it into a simpler one corresponding to the martingale case thanks to the artificial extension method. Let us consider the hedging numéraire $\widetilde{a}$ and the associated $\widetilde{a}$-extended assets family $\left\{V(\widetilde{a}), S^{0}, S\right\}$. We can define the equivalent $\widetilde{a}$-martingale measure $\widetilde{P}(\widetilde{a})$ given by the relation

$$
\frac{d \widetilde{P}(\widetilde{a})}{d P}=\frac{V_{T}(\widetilde{a})^{2}}{\mathbb{E}\left[V_{T}(\widetilde{a})\right]^{2}}
$$

and we call it the variance-optimal $\widetilde{a}$-martingale measure. Let us consider the quadratic optimization problem

$$
J^{\widetilde{a}}(x, F)=\min _{\phi(\widetilde{a}) \in \Phi(\widetilde{a})} \mathbb{E}_{\widetilde{P}(\widetilde{a})}\left[\frac{F}{V_{T}(\widetilde{a})}-x-\int_{0}^{T} \phi_{t}(\widetilde{a}) d X_{t}(\widetilde{a})\right]^{2} \quad\left(\mathcal{H}^{\widetilde{a}}(x)\right)
$$

A straightforward extension of Proposition 5.1 in GLP gives that problems $(\mathcal{H}(x))$ and $\left(\mathcal{H}^{\widetilde{a}}(x)\right)$ are equivalent in the following sense: if $\theta^{*}$ and $\phi^{*}(\widetilde{a})$ are the unique solutions of, respectively, problem $(\mathcal{H}(x))$ and problem $\left(\mathcal{H}^{\widetilde{a}}(x)\right)$, then they have the same value process, i.e.

$$
S_{t}^{0}\left(V_{0}+\int_{0}^{t} \vartheta_{s}^{*} d\left(S / S^{0}\right)_{s}\right)=V_{t}(\widetilde{a})\left(V_{0}+\int_{0}^{t} \phi_{s}^{*}(\widetilde{a}) d X_{s}(\widetilde{a})\right), \quad t \in[0, T] .
$$

Moreover, the relation (5.2) in GLP, between their minimal quadratic risks, is still verified, i.e.

$$
J(x, F)=\mathbb{E}\left[V_{T}(\widetilde{a})\right]^{2} J^{\widetilde{a}}(x, F) .
$$

Now since $\widetilde{P}(\widetilde{a}) \in \mathcal{M}_{2}^{e}(\widetilde{a})$, the continuous process $X(\widetilde{a})$ is a locally square integrable martingale under $\widetilde{P}(\widetilde{a})$. Furthermore, being $F$ square integrable under $P$, the claim $F / V_{T}(\widetilde{a})$ is square integrable under $\widetilde{P}(\widetilde{a})$. The infinite-dimensional GKW-projection theorem (Proposition 4) implies that there exists a $\mathcal{U}$-valued predictable process $\phi^{F}(\widetilde{a})$ satisfying

$$
\mathbb{E}_{\widetilde{P}(\widetilde{a})}\left[\left\langle\int \phi^{F}(\widetilde{a}) d X(\widetilde{a})\right\rangle_{T}\right]<\infty
$$

and a real-valued square integrable $\widetilde{P}(\widetilde{a})$-martingale $\widetilde{R}(\widetilde{a})$, orthogonal to $X(\widetilde{a})$ under $\widetilde{P}(\widetilde{a})$, such that

$$
\frac{F}{V_{T}(\widetilde{a})}=\mathbb{E}_{\widetilde{P}(\widetilde{a})}\left[\frac{F}{V_{T}(\widetilde{a})}\right]+\int_{0}^{T} \phi^{F}(\widetilde{a}) d X(\widetilde{a})+\widetilde{R}_{T}(\widetilde{a}) .
$$


Clearly, the solution $\phi^{*}(\widetilde{a})$ to problem $\left(\mathcal{H}^{\widetilde{a}}(x)\right)$ is given by the integrand in the decomposition (17), i.e. $\phi^{*}(\widetilde{a})=\phi^{F}(\widetilde{a})$, and the associated minimal quadratic risk of problem $\left(\mathcal{H}^{\widetilde{a}}(x)\right)$ is given by

$$
J^{\widetilde{a}}(x, F)=\left(\mathbb{E}_{\widetilde{P}(\widetilde{a})}\left[\frac{F}{V_{T}(\widetilde{a})}\right]-x\right)^{2}+\mathbb{E}_{\widetilde{P}(\widetilde{a})}\left[\widetilde{R}_{T}(\widetilde{a})\right]^{2} .
$$

We now summarize how to "theoretically" solve our initial infinite-dimensional MVHproblem $(\mathcal{H}(x))$ : compute the hedging numéraire $\widetilde{a}$ and consider the MVH-problem $\left(\mathcal{H}^{\widetilde{a}}(x)\right)$ corresponding to the price process $X(\widetilde{a})$, the strategies set $\Phi(\widetilde{a})$ and the probability $\widetilde{P}(\widetilde{a})$, which is a martingale measure for the new integrator; the GKW-projection theorem gives its unique solution $\phi^{*}(\widetilde{a})$. Now, in order to find the optimal strategy $\vartheta^{*}$, solve with respect to $\vartheta$ the following stochastic equation:

$$
S_{t}^{0}\left(V_{0}+\int_{0}^{t} \vartheta_{s} d\left(S / S^{0}\right)_{s}\right)=V_{t}(\widetilde{a})\left(V_{0}+\int_{0}^{t} \phi_{s}^{*}(\widetilde{a}) d X_{s}(\widetilde{a})\right), \quad t \in[0, T] .
$$

Observe that Proposition 9 ensures the existence of a solution for this equation.

We conclude this section by considering the problem

$$
\min _{x \in \mathbb{R}} J(x, F),
$$

which corresponds to the projection of $F$ on the closed subspace $\left\{G_{T}(x, \Theta): x \in \mathbb{R}\right\}$ of $L^{2}(P)$ (to see this use the same argument as in GLP, p. 195). The solution $x^{*}(F)$ to problem $(\mathcal{H})$ is called the approximation price for $F$ (see Schweizer [22]) and is a generalization of the usual arbitrage-free price for $F$. From (16), (18) and Proposition 8 one can easily deduce that

$$
x^{*}(F)=\mathbb{E}_{\widetilde{P}}\left[\frac{F}{S_{T}^{0}}\right],
$$

so that, even in this setting, the VOMM can be interpreted as a viable price system corresponding to a mean-variance criterion.

\section{Finite-dimensional MVH problems}

Let $n \geq 1$ be given. We denote by $\mathbb{F}^{n}=\left\{\mathcal{F}_{t}^{n}: t \in[0, T]\right\}$ the (completed) filtration generated by the $n$-dimensional primitive assets family $\left\{S^{0}, \bar{S}^{n}\right\}$ where $\bar{S}^{n}=\left(S^{1}, \ldots, S^{n}\right)$, $\mathcal{F}^{n}=\mathcal{F}_{T}^{n}$, by $P^{n}$ the restriction on $\mathcal{F}^{n}$ of the probability measure $P$ and we set

$$
\begin{aligned}
\mathcal{M}_{2}^{n}= & \left\{Q^{n} \text { probability measure on } \mathcal{F}^{n}: Q^{n} \ll P^{n},\right. \\
& \left.\frac{1}{S_{T}^{0}} \frac{d Q^{n}}{d P} \in L^{2}(P), \bar{S}^{n} / S^{0} \text { is a local } Q^{n} \text {-martingale }\right\}
\end{aligned}
$$

and

$$
\mathcal{M}_{2}^{n, e}=\left\{Q^{n} \in \mathcal{M}_{2}^{n}: Q^{n} \sim P^{n}\right\}
$$


Assumption (6) ensures that, for all $n \geq 1$, the set $\mathcal{M}_{2}^{n, e}$ is not empty.

Consider the following $n$-dimensional mean-variance hedging $(n-\mathrm{MVH})$ problem for a given contingent claim $F \in L^{2}(P)$ and an initial endowment $x \in \mathbb{R}$ :

$$
\min _{\vartheta^{n} \in \Theta^{n}} \mathbb{E}\left[F-S_{T}^{0}\left(x+\int_{0}^{T} \vartheta_{t}^{n} d\left(\bar{S}^{n} / S^{0}\right)_{t}\right)\right]^{2},
$$

where $\Theta^{n}$ denotes the set of all $\mathbb{R}^{n}$-valued $\bar{S}^{n} / S^{0}$-integrable $\mathbb{F}^{n}$-predictable processes $\vartheta^{n}$ such that $S_{T}^{0} \int_{0}^{T} \vartheta_{t}^{n} d\left(\bar{S}^{n} / S^{0}\right)_{t} \in L^{2}\left(\mathcal{F}^{n}, P\right)$ and for all $Q^{n} \in \mathcal{M}_{2}^{n}$, the process $\int \vartheta^{n} d\left(\bar{S}^{n} / S^{0}\right)$ is a $Q^{n}$-martingale. All the objects we have introduced in the previous two sections have their $n$-dimensional counterparts, their notation and financial interpretations will be selfevident.

The aim of this section is to study the asymptotical behavior, as $n \rightarrow \infty$, of the sequence $\left(\vartheta^{n, *}\right)_{n \geq 1}$, where $\vartheta^{n, *}$ is the unique solution to problem $\left(\mathcal{H}_{n}(x)\right)$.

Let us consider the following finite-dimensional dual problem associated to the assets family $\left\{S^{0}, \bar{S}^{n}\right\}, n \geq 1$ :

$$
\min _{Q^{n} \in \mathcal{M}_{2}^{n}} \mathbb{E}\left[\frac{1}{S_{T}^{0}} \frac{d Q^{n}}{d P^{n}}\right]^{2}
$$

Under the standing assumption (6), problem $\left(\mathcal{D}_{n}\right)$ admits a unique solution $\widetilde{P}^{n} \in \mathcal{M}_{2}^{n}$, which we call $n$-dimensional variance-optimal martingale measure (abbr. $n$-VOMM).

Following Delbaen and Schachermayer [7], we denote $K_{0}^{n}$ the subspace of $L^{\infty}\left(\mathcal{F}^{n}, P\right)$ spanned by the stochastic integrals of the form

$$
f_{n}=h_{n}^{\prime}\left(\left(\bar{S}^{n} / S^{0}\right)_{\tau_{2}}-\left(\bar{S}^{n} / S^{0}\right)_{\tau_{1}}\right)
$$

where $\tau_{1} \leq \tau_{2}$ are $\mathbb{F}^{n}$-stopping times such that the stopped process $\left(\bar{S}^{n} / S^{0}\right)^{\tau_{2}}$ is bounded and $h_{n}$ is a bounded $\mathbb{R}^{n}$-valued $\mathcal{F}_{\tau_{1}}^{n}$-measurable function.

We denote by $\widehat{K_{0}^{n}}$ the closure of $K_{0}^{n}$ in $L^{2}(P)$ and set $\widehat{K^{n}}:=\overline{\operatorname{span}\left(K_{0}^{n}, 1\right)}$. Furthermore, $\widehat{K_{0}}$ denotes the closure of $K_{0}:=\cup_{n \geq 1} K_{0}^{n} \subset L^{\infty}\left(\mathcal{F}^{n}, P\right)$ in $L^{2}(P)$ and $\widehat{K}=\overline{\operatorname{span}\left(K_{0}, 1\right)}$.

Assuming (6), we deduce from Lemma 2.1(c) in [7] the following characterizations of $\widetilde{P}$ and $\widetilde{P}^{n}$ (here we identify any measure $Q$ with the linear functional $\mathbb{E}_{Q}[\cdot]$ and linear functionals on $L^{2}(P)$ with elements of $\left.L^{2}(P)\right)$ :

$\widetilde{P}\left(\right.$ resp. $\left.\widetilde{P}^{n}\right)$ is the unique element of $\widehat{K}$ (resp. $\widehat{K^{n}}$ ) vanishing on $\widehat{K_{0}}$ (resp. $\widehat{K_{0}^{n}}$ ) and equaling 1 on the constant function 1.

Given this characterization, an immediate application of the projection theorem for Hilbert spaces leads to the following

Proposition 11 Assume (6). The sequence $\widetilde{P}^{n}$ converges in $L^{2}(P)$, as $n \rightarrow \infty$, to the VOMM $\widetilde{P}$ solution to problem $(\mathcal{D})$. 
Now, we consider the following $n$-MVH problem:

$$
\min _{\vartheta^{n} \in \Theta^{n}} \mathbb{E}\left[S_{T}^{0}\left(1+\int_{0}^{T} \vartheta_{t}^{n} d\left(\bar{S}^{n} / S^{0}\right)_{t}\right)\right]^{2}
$$

and we set

$$
G_{T}^{n}\left(x, \Theta^{n}\right):=\left\{S_{T}^{0}\left(x+\int_{0}^{T} \vartheta_{s}^{n} d\left(\bar{S}^{n} / S^{0}\right)_{s}\right): \vartheta^{n} \in \Theta^{n}\right\} .
$$

Under (6), each problem $\left(\mathcal{P}_{n}\right)$ has a solution $\widetilde{a}^{n} \in \Theta^{n}$, which leads to a unique optimal terminal wealth $V_{T}^{n}\left(\widetilde{a}^{n}\right)=V_{T}^{1, \widetilde{a}^{n}}=S_{T}^{0}\left(1+\int_{0}^{T} \widetilde{a}_{t}^{n} d\left(\bar{S}^{n} / S^{0}\right)_{t}\right)$. As in GLP and in the previous section, one has $V^{n}\left(\widetilde{a}^{n}\right)>0$ a.s., so that one can use it as a numéraire called $n$-dimensional hedging numéraire. We define the $n$-dimensional variance-optimal $\widetilde{a}^{n}$-martingale measure by the relation

$$
\frac{d \widetilde{P}^{n}\left(\widetilde{a}^{n}\right)}{d P^{n}}=\frac{V_{T}^{n}\left(\widetilde{a}^{n}\right)^{2}}{\mathbb{E}_{n}\left[V_{T}^{n}\left(\widetilde{a}^{n}\right)\right]^{2}}
$$

and consider the $n$-dimensional analogue of problem $\left(\mathcal{H}^{\widetilde{a}}(x)\right)$ :

$$
\min _{\phi^{n}\left(\widetilde{a}^{n}\right) \in \Phi^{n}\left(\widetilde{a}^{n}\right)} \mathbb{E}_{\widetilde{P}^{n}\left(\widetilde{a}^{n}\right)}\left[\frac{F}{V_{T}^{n}\left(\widetilde{a}^{n}\right)}-x-\int_{0}^{T} \phi_{t}^{n}\left(\widetilde{a}^{n}\right) d{\overline{X_{t}\left(\widetilde{a}^{n}\right)}}^{n}\right]^{2} \quad\left(\mathcal{H}_{n}^{\widetilde{a}^{n}}(x)\right)
$$

where ${\overline{X\left(\widetilde{a}^{n}\right)}}^{n}=\bar{X}^{n} / V^{n}\left(\widetilde{a}^{n}\right)$ and $\Phi^{n}\left(\widetilde{a}^{n}\right)$ is the set of all $\mathbb{R}^{n+1}$-valued $\overline{X\left(\widetilde{a}^{n}\right)^{n}}$-integrable predictable processes such that $V_{T}^{n}\left(\widetilde{a}^{n}\right) \int_{0}^{T} \phi_{t}^{n}\left(\widetilde{a}^{n}\right) d{\overline{X_{t}\left(\widetilde{a}^{n}\right)}}^{n} \in L^{2}\left(\mathcal{F}^{n}, P\right)$ and for all $Q^{n}\left(\widetilde{a}^{n}\right) \in$ $\mathcal{M}_{2}^{n}\left(\widetilde{a}^{n}\right)$, the process $\int \phi^{n}\left(\widetilde{a}^{n}\right) d \overline{X\left(\widetilde{a}^{n}\right)^{n}}$ is a local $Q^{n}\left(\widetilde{a}^{n}\right)$-martingale.

We recall that, for all $n \geq 1$, the solution to problem $\left(\mathcal{H}_{n}^{\widetilde{a}^{n}}(x)\right)$ is given by the $\mathbb{R}^{n+1}$ valued predictable integrand $\phi^{n, *}\left(\widetilde{a}^{n}\right)$ satisfying the integrability condition

$$
\mathbb{E}_{\widetilde{P}^{n}\left(\widetilde{a}^{n}\right)}\left[\left\langle\int \phi^{n, *}\left(\widetilde{a}^{n}\right) d{\overline{X\left(\widetilde{a}^{n}\right)^{n}}}^{n}\right\rangle_{T}\right]<\infty
$$

in the following GKW-decomposition

$$
\frac{F}{V_{T}^{n}\left(\widetilde{a}^{n}\right)}=\mathbb{E}_{\widetilde{P}^{n}\left(\widetilde{a}^{n}\right)}\left[\frac{F}{V_{T}^{n}\left(\widetilde{a}^{n}\right)}\right]+\int_{0}^{T} \phi^{n, *}\left(\widetilde{a}^{n}\right) d \overline{X\left(\widetilde{a}^{n}\right)^{n}}+\widetilde{R}_{T}^{n}\left(\widetilde{a}^{n}\right),
$$

where $\widetilde{R}^{n}\left(\widetilde{a}^{n}\right)$ is a real-valued square integrable $\left(\mathbb{F}^{n}, \widetilde{P}^{n}\left(\widetilde{a}^{n}\right)\right)$-martingale, orthogonal to $\overline{X\left(\widetilde{a}^{n}\right)}{ }^{n}$ under $\widetilde{P}^{n}\left(\widetilde{a}^{n}\right)$.

Proposition 12 Let $\vartheta^{n, *}$ and $\phi^{n, *}$ be solutions to problems (respectively) $\left(\mathcal{H}_{n}(x)\right.$ ) and $\left(\mathcal{H}_{n}^{\widetilde{a}^{n}}(x)\right)$ for all $n \geq 1$. Then we have the following assertions:

1. $\int_{0}^{T} \vartheta_{t}^{n, *} d\left(\bar{S}^{n} / S^{0}\right)_{t}$ converges to $\int_{0}^{T} \vartheta_{t}^{*} d\left(S / S^{0}\right)_{t}$ in $L^{2}(P)$ as $n \rightarrow \infty$, where $\vartheta^{*}$ is the solution to problem $(\mathcal{H}(x))$; 


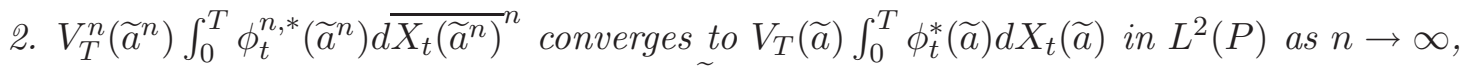
where $\phi^{*}$ is the solution to problem $\left(\mathcal{H}^{\widetilde{a}}(x)\right)$.

Proof. 1. It suffices to note that for all $n \geq 1, S_{T}^{0} \int_{0}^{T} \vartheta_{t}^{n, *} d\left(\bar{S}^{n} / S^{0}\right)_{t}$ is the orthogonal projection of $S_{T}^{0} \int_{0}^{T} \vartheta_{t}^{*} d\left(S / S^{0}\right)_{t}$ onto the subspace $G_{T}^{n}\left(0, \Theta^{n}\right)$ closed in $L^{2}(P)$.

2. By Proposition 3.2 of GLP and Proposition 9 we have, respectively, that for all $n \geq 1$

$$
S_{T}^{0}\left(x+\int_{0}^{T} \vartheta_{t}^{n, *} d\left(\bar{S}^{n} / S^{0}\right)_{t}\right)=V_{T}^{n}\left(\widetilde{a}^{n}\right)\left(x+\int_{0}^{T} \phi_{t}^{n, *}\left(\widetilde{a}^{n}\right) d \bar{X}_{t}\left(\widetilde{a}^{n}\right)^{n}\right)
$$

and

$$
S_{T}^{0}\left(x+\int_{0}^{T} \vartheta_{t}^{*} d\left(S / S^{0}\right)_{t}\right)=V_{T}(\widetilde{a})\left(x+\int_{0}^{T} \phi_{t}^{*}(\widetilde{a}) d X_{t}(\widetilde{a})\right) .
$$

Then assertion 2. follows easily.

Remark 13 We point out that, with respect to the Emery topology, used to prove Proposition 9, the $L^{2}(P)$-convergence works only for the finite-dimensional optimal hedging strategies. Then, to establish the correspondence between the sets $\Theta$ and $\Phi(a)$ of all strategies, one has to deal with convergence in $\mathcal{S}(P)$.

\section{References}

[1] Ansel, J.P. and Stricker, C. (1993): Décomposition de Galtchouk-Kunita-Watanabe. Séminaire de Probabilités XXVII, Lecture Notes in Math., 1557, 30-32.

[2] Bjork, T., Näslund, B. (1998): Diversified Portfolios in Continuous Time. European Finance Review, 1998, 361-387.

[3] Bouleau, N. and D. Lamberton (1989): Residual Risks and Hedging Strategies in Markovian Markets. Stoch. Process. Appl. 33, 131-150.

[4] De Donno, M. (2004): A note on completeness in large financial markets. Math. Finance 14, no. 2, 295-315.

[5] De Donno, M., Guasoni, P., Pratelli, M. (2005): Super-replication and utility maximization in large financial markets. Stochastic Process. Appl. 115, no. 12, 2006-2022.

[6] De Donno, M. and M. Pratelli (2003): Stochastic Integration with respect to a sequence of semimartingales. To appear in Sém. Probabilités. Downloadable at: http://www.dm.unipi/ mdedonno.papers.html.

[7] Delbaen, F. and W. Schachermayer (1996a): The Variance-Optimal Martingale Measure for Continuous Processes. Bernoulli 2(1), 81-105.

[8] Delbaen, F. and Schachermayer, W. (1996b): Attainable Claims with p'th Moments. Annales de l'I.H.P., 32 (6), 743-763. 
[9] Dellacherie, C. (1978): Quelques applications du lemme de Borel-Cantelli à la théorie des semimartingales. Séminaire de Probabilités XII, Lecture Notes in Math., 649, 742745 .

[10] Emery, M. (1979): Une topologie sur l'espace des semi-martingales. Séminaire de Probabilités XIII, Lecture Notes in Math., 721, 260-280.

[11] Föllmer, H. and D. Sondermann (1986): Hedging of Non-Redundant Contingent Claims. In "Contributions to Mathematical Economics", (Mas-Collel A. and Hildebrand W. Editors). Amsterdam: North Holland, 205-223.

[12] Gourieroux, C., J.P. Laurent, and H. Pham (1998): Mean-variance hedging and numéraire. Mathematical Finance, 8 (3), 179-200.

[13] Jacod, J. (1979): Calcul Stochastique et problème de martingales, Lectures Notes in Math., 714.

[14] Kabanov, Yu. M. and D.O. Kramkov (1994): Large financial markets: asymptotic arbitrage and contiguity. Theory Probab. Appl. 39(1), 222-229.

[15] Kabanov, Yu. M. and D.O. Kramkov (1998): Asymptotic arbitrage in large financial markets. Finance and Stoch. 2, 143-172.

[16] Klein, I. and W. Schachermayer (1996): Asymptotic Arbitrage in Non-Complete Large Financial Markets. Theory Probab. Appl. 41 (4), 780-788.

[17] Kreps, D. (1981): Arbitrage and Equilibrium in Economies with Infinetely Many Commodities. Journal of Math. Econ. 8, 15-35.

[18] Memin, J. (1980): Espaces de semi-martingales et changement de probabilité. $Z$. Wahrscheinlichkeitstheorie verw. Gebiete, 52, 9-39.

[19] Mikulevicius, R. and B.L. Rozovskii (1998): Normalized Stochastic Integrals in Topological Vector Spaces. In Séminaire de Probabilités XXXIII. Lecture notes in Mathematics, Springer Verlag, New York.

[20] Protter, P. (1980). Stochastic Integration and Differential Equations. Berlin, New York: Springer Verlag.

[21] Rheinländer, T. and M. Schweizer (1997): On $L^{2}$-projections on a space of stochastic integrals. Ann. Probab. 25, 1810-1831.

[22] Schweizer, M. (1996): Approximation Pricing and the Variance-Optimal Martingale Measure. Ann. Probab. 64, 206-236. 